\title{
Criminologie
}

\section{Perspectives néo-zélandaises sur la justice des mineurs au Canada}

\section{Allison Morris et Gabrielle Maxwell}

Volume 32, numéro 2, automne 1999

La justice des mineurs

URI : https://id.erudit.org/iderudit/004736ar

DOI : https://doi.org/10.7202/004736ar

Aller au sommaire du numéro

\section{Éditeur(s)}

Les Presses de l'Université de Montréal

ISSN

0316-0041 (imprimé)

1492-1367 (numérique)

Découvrir la revue

Citer cet article

Morris, A. \& Maxwell, G. (1999). Perspectives néo-zélandaises sur la justice des mineurs au Canada. Criminologie, 32(2), 37-54. https://doi.org/10.7202/004736ar

\section{Résumé de l'article}

Le gouvernement canadien a présenté des propositions de changement de la justice des mineurs qui mettent l'accent sur la prévention de la délinquance des jeunes; sur la déjudiciarisation et la réduction de la mise sous garde ; sur le fait de tenir les jeunes responsables de leurs infractions de manière significative; sur l'établissement de programmes qui produisent des effets à l'endroit des contrevenants, particulièrement les délinquants multirécidivistes et violents ; sur le fait de rendre la justice des adolescents mieux adaptée aux différences culturelles; sur l'accroissement de la responsabilité des parents; et sur le fait de donner une voix plus forte aux victimes. Nombre de ces thèmes sont également présents dans le régime de justice des mineurs introduit en Nouvelle-Zélande en 1989.L'article fait part de l'expérience néo-zélandaise de déjudiciarisation des jeunes réalisée en engageant les jeunes et leurs familles dans des conférences familiales qui comportent une rencontre avec la victime et où se décide comment réagir à l'infraction. La recherche néo-zélandaise montre que les conférences familiales peuvent être efficaces pour tenir les jeunes responsables de leurs actes, pour éviter la judiciarisation et la mise sous garde, pour offrir des options culturellement adéquates, pour encourager la responsabilité parentale, pour donner un rôle aux victimes et pour réduire la récidive. Il est suggéré que le Canada pourrait adapter des aspects du régime néo-zélandais pour développer des stratégies conformes à ses objectifs.
Ce document est protégé par la loi sur le droit d'auteur. L'utilisation des services d'Érudit (y compris la reproduction) est assujettie à sa politique d'utilisation que vous pouvez consulter en ligne.

https://apropos.erudit.org/fr/usagers/politique-dutilisation/ 


\title{
Perspectives néo-zélandaises sur la justice des mineurs au Canada
}

\author{
Allison Morris \\ Chercheure \\ Institut de criminologie \\ Université Victoria de Wellington - Nouvelle-Zélande \\ amm7@hermes.cam.ac.uk \\ Gabrielle Maxwell \\ Professeure \\ Institut de criminologie \\ Université Victoria de Wellington - Nouvelle-Zélande \\ gabrielle.maxwell@vuw.ac.nz
}

RÉSumé - Le gouvernement canadien a présenté des propositions de changement de la justice des mineurs qui mettent l'accent sur la prévention de la délinquance des jeunes; sur la déjudiciarisation et la réduction de la mise sous garde ; sur le fait de tenir les jeunes responsables de leurs infractions de manière significative ; sur l'établissement de programmes qui produisent des effets à l'endroit des contrevenants, particulièrement les délinquants multirécidivistes et violents; sur le fait de rendre la justice des adolescents mieux adaptée aux différences culturelles ; sur l'accroissement de la responsabilité des parents; et sur le fait de donner une voix plus forte aux victimes. Nombre de ces thèmes sont également présents dans le régime de justice des mineurs introduit en Nouvelle-Zélande en 1989.

L'article fait part de l'expérience néo-zélandaise de déjudiciarisation des jeunes réalisée en engageant les jeunes et leurs familles dans des conférences familiales qui comportent une rencontre avec la victime et où se décide comment réagir à l'infraction. La recherche néo-zélandaise montre que les conférences familiales peuvent être efficaces pour tenir les jeunes responsables de leurs actes, pour éviter la judiciarisation et la mise sous garde, pour offrir des options culturellement adéquates, pour encourager la responsabilité parentale, pour donner un rôle aux victimes et pour réduire la récidive. Il est suggéré que le Canada pourrait adapter des aspects du régime néo-zélandais pour développer des stratégies conformes à ses objectifs. 
ABStRACt - Canada is considering new proposals for juvenile justice with emphasis on the prevention of juvenile crime ; keeping young people out of courts and custody ; holding young people accountable for their offences in meaningful ways ; providing effective programs to deal with offenders, especially repeat and violent offenders ; making the juvenile justice system more culturally responsive ; increasing the responsibility of parents ; and giving victims a greater voice. Many of these issues have been taken into account by the juvenile justice system introduced in New Zealand in 1989.

This paper comments on the New Zealand experience of diverting young people from courts by involving them and their families in family group conferences where they meet with victims to decide on how best to respond to the offences. New Zealand research demonstrates that family group conferences can be effective ways of holding young people accountable, diverting them from courts and custodial sentences, providing for culturally appropriate options, encouraging parental responsibility, giving victims a role and reducing repeat offences. It is suggested that Canada could adapt certain aspects of the New Zealand system in developing new strategies to achieve its objectives.

\section{Introduction}

Actuellement, plusieurs pays réévaluent leur système de justice des mineurs. Presque partout, on pense que la délinquance juvénile est en recrudescence et que les mineurs commettent des délits plus graves à un plus jeune âge. Que ces opinions soient ou non exactes n'est pas vraiment au cœur du débat. En fait, le public a perdu confiance dans la gestion de la justice des mineurs et les politiciens éprouvent le besoin de « durcir » leurs positions à l'égard des jeunes délinquants.

Au Canada, le gouvernement fédéral annonça en mai 1998 la mise au point d'une stratégie destinée à « renouveler le système de justice des mineurs » (Ministère de la Justice du Canada, 1998 ; Thomson, 1998). Au moment d'écrire cet article, la présentation d'un projet de loi sur la question était toujours attendue au Parlement canadien. Les changements proposés dans ce projet semblent obéir à plusieurs préoccupations : le besoin de prévenir la délinquance juvénile et d'éviter à des jeunes la judiciarisation et la mise sous garde ; le besoin de responsabiliser les jeunes face à leurs délits en utilisant des méthodes qui ont du sens ; le développement de programmes destinés à traiter de manière efficace les jeunes délinquants, spécialement ceux qui ont récidivé et ont agi avec violence ; le besoin de rendre la justice des mineurs plus proche des valeurs culturelles; le besoin d'augmenter la responsabilité des parents et de donner une voix aux victimes. 
Commenter des propositions de lois faites dans un pays étranger peut sembler dangereux ; les chercheurs accomplissant ce travail peuvent en effet être critiqués parce qu'ils ne peuvent apprécier complètement les nuances que recouvrent ces changements. Cependant, les propositions canadiennes font plus que susciter le simple étonnement et appellent une analyse plus profonde quand elles sont abordées selon une perspective néo-zélandaise. On peut notamment s'interroger sur le rôle central assigné aux tribunaux de la jeunesse alors que les processus de prise de décision alternatifs tels que les conférences familiales sont confinés à un rôle marginal. On peut aussi s'interroger sur la valeur du sentiment d'humiliation engendré par la publication du nom de jeunes délinquants.

Dans cet article, nous commenterons brièvement ces deux questions pour ensuite présenter plus en détail l'expérience des conférences familiales en Nouvelle-Zélande. Par ce biais, nous espérons montrer comment certains des objectifs visés par les propositions canadiennes pourraient être plus facilement atteints. Parmi ces objectifs, citons l'établissement de mesures de rechange à la judiciarisation et à la mise sous garde des mineurs ; la responsabilisation des jeunes face à leurs délits ; l'adaptation du système de justice des mineurs au contexte culturel ; la plus grande responsabilisation des parents et l'importance accrue accordée aux victimes. Puisque, d'après les propositions canadiennes, le système de justice des mineurs a pour but principal de protéger la société, nous soulignerons aussi à quel point les conférences familiales peuvent contribuer à la réduction de la récidive. En développant ces questions, nous utiliserons les recherches que nous avons menées au sein du système néo-zélandais ${ }^{1}$.

1. D'août 1990 à mai 1991, assistées d'une équipe de dix chercheurs, nous avons suivi ce qui est arrivé à environ 700 jeunes reconnus pour avoir commis des délits dans une des cinq régions du pays (Maxwell et Morris, 1993). Là où c'était possible, nous assistions aux conférences familiales qui touchèrent un peu plus de 200 jeunes; nous interviewions les jeunes, leurs familles, les victimes et les professionnels impliqués. Lorsque les jeunes avaient fait l'objet d'une arrestation (ce qui était le cas pour 70 d'entre eux), nous interrogions le policier qui avait procédé à l'arrestation et enquêtions sur ce qui s'était passé au tribunal de la jeunesse. Par la suite, nous avons recherché ce qui était arrivé à la plupart de ces jeunes, en interrogeant les familles et en vérifiant les dossiers de ces jeunes trois ou six mois après notre premier contact. Nous avons aussi recueilli des statistiques significatives à partir de bases de données nationales; nous avons compilé les statistiques tirées des dossiers de police, des services sociaux et des tribunaux. Par la suite, à partir de 1997, nous avons essayé de retrouver et de réinterroger ces jeunes âgés de 14 à 17 ans qui avaient pris part aux conférences familiales durant notre recherche de 1990-1991. Nous les avons questionnés sur leur enfance, leurs souvenirs des conférences familiales et leur vie après ces conférences. Nous avons aussi fait des recherches sur leur profil délinquant en nous basant sur leurs nouvelles condamnations et sur les enquêtes de délinquance autorévélée (Morris et al., 1998). 


\section{Le rôle central accordé aux tribunaux dans la gestion de la délinquance juvénile}

Même si elles encouragent le recours à des mesures de rechange, les propositions canadiennes continuent à accorder une place centrale aux tribunaux de la jeunesse. À l'opposé, de nombreux pays ne considèrent plus désormais les tribunaux comme des lieux majeurs de prise de décision, spécialement en ce qui a trait aux jeunes délinquants. Les corps policiers évitent souvent la judiciarisation des mineurs en les dirigeant vers d'autres instances, comme les conférences familiales ou d'autres procédures de rechange. Un tel changement s'explique principalement par le fait que les tribunaux ne sont plus considérés comme le meilleur recours pour amener les jeunes, leurs familles, les victimes et la communauté à s'impliquer. Le caractère formel que revêtent les tribunaux a certes plusieurs « avantages » : il peut conférer une autorité au processus, il contribue à maintenir la dignité de la loi et peut souligner le caractère sérieux de l'événement. Cependant, ce caractère formel compte aussi plusieurs «inconvénients ». Il peut augmenter la distance que l'on ressent par rapport aux professionnels, incluant ceux qui y ont un pouvoir de décision. Les "véritables » parties impliquées dans le délit - les jeunes délinquants, leurs familles, leurs groupes d'appartenance et les victimes - peuvent se sentir dépossédées de tout pouvoir, exclues et aliénées. Pour beaucoup de personnes passées devant les tribunaux, l'aspect formel de ces derniers soutient des traditions qui à leurs yeux n'ont pas de sens et représentent un rituel monoculturel; il peut rendre les procédures incompréhensibles aux parties "réellement » concernées. Une autre raison du recours à des processus autres que judiciaires tient au fait que peu de jeunes nient leur responsabilité à l'endroit de leurs actes ; dès lors, les tribunaux, qui sont le forum le plus approprié pour mener des procès, ont à tenir un rôle différent, celui d'imposer des peines. Or, les tribunaux ne sont justement pas les mieux habilités pour accomplir une telle tâche.

\section{L'impact de l'humiliation liée à la publication des noms}

Le travail théorique mené par Braithwaite (1989) sur l'humiliation (shaming) établit une distinction entre l'humiliation réintégrative qui favorise le retour accepté du délinquant dans la communauté et l'humiliation stigmatisante qui est plutôt un facteur d'exclusion. Cependant, 
nous ne disposons pas de preuves suffisantes pour affirmer que l'humiliation constitue un prélude nécessaire à la réintégration. De plus, selon des études psychologiques (Tangney et al., 1996), nous savons que l'humiliation peut affecter l'estime de soi et peut favoriser la colère et l'agressivité. Dans nos propres recherches (Maxwell, 1998 ; Morris et al., 1998), nous avons aussi remarqué que les jeunes persistant dans la récidive et leurs parents étaient plus nombreux que les non-récidivistes à témoigner de sentiments de malaise au sujet d'eux-mêmes éprouvés au sortir des conférences familiales. Bien qu'en l'occurrence il ne s'agisse que d'une procédure exclusivement privée, ces conférences avaient néanmoins suscité un profond sentiment de honte. Dans leur ensemble, toutes ces recherches remettent en question la valeur de telles pratiques : rendre publics les noms des jeunes délinquants peut en effet s'avérer contre-productif. En d'autres termes, on court réellement de grands risques quand on fait naître délibérément un sentiment de honte.

\section{Un survol du système de justice des mineurs en Nouvelle-Zélande}

\section{Cheminement à travers le système}

En Nouvelle-Zélande, le système de justice des mineurs s'occupe d'enfants et de jeunes qui sont âgés d'au moins 10 ans et qui avaient moins de 17 ans au moment où ils ont commis un délit, à l'exception de ceux qui ont commis un meurtre ou un homicide involontaire coupable. Les délits très graves comme le viol, le vol et les voies de fait sont traités, du moins au départ, dans le cadre de la justice des mineurs. Parmi ces types de délit, Maxwell et Morris (1993) citent comme exemples le cambriolage d'une maison et le viol d'une jeune femme, l'incendie volontaire et la destruction de tout un bâtiment scolaire, le fait d'avoir frappé une victime à la tête durant un cambriolage et des voies de faits à la suite desquelles la victime a subi des séquelles permanentes au cerveau ${ }^{2}$.

La figure 1 décrit les avenues possibles dans le système de justice des mineurs en Nouvelle-Zélande.

2. Ultérieurement, certains jeunes délinquants sont transférés du Tribunal de la jeunesse au Tribunal de district (pour adultes) ou à la Cour supérieure suivant la gravité du délit et la « carrière délinquante » du jeune. Mais de tels transferts sont rares et la grande majorité des mineurs délinquants est prise en charge par le système de justice des mineurs. 


\section{F I G URE 1}

Cheminement à travers le système de justice des mineurs en Nouvelle-Zélande

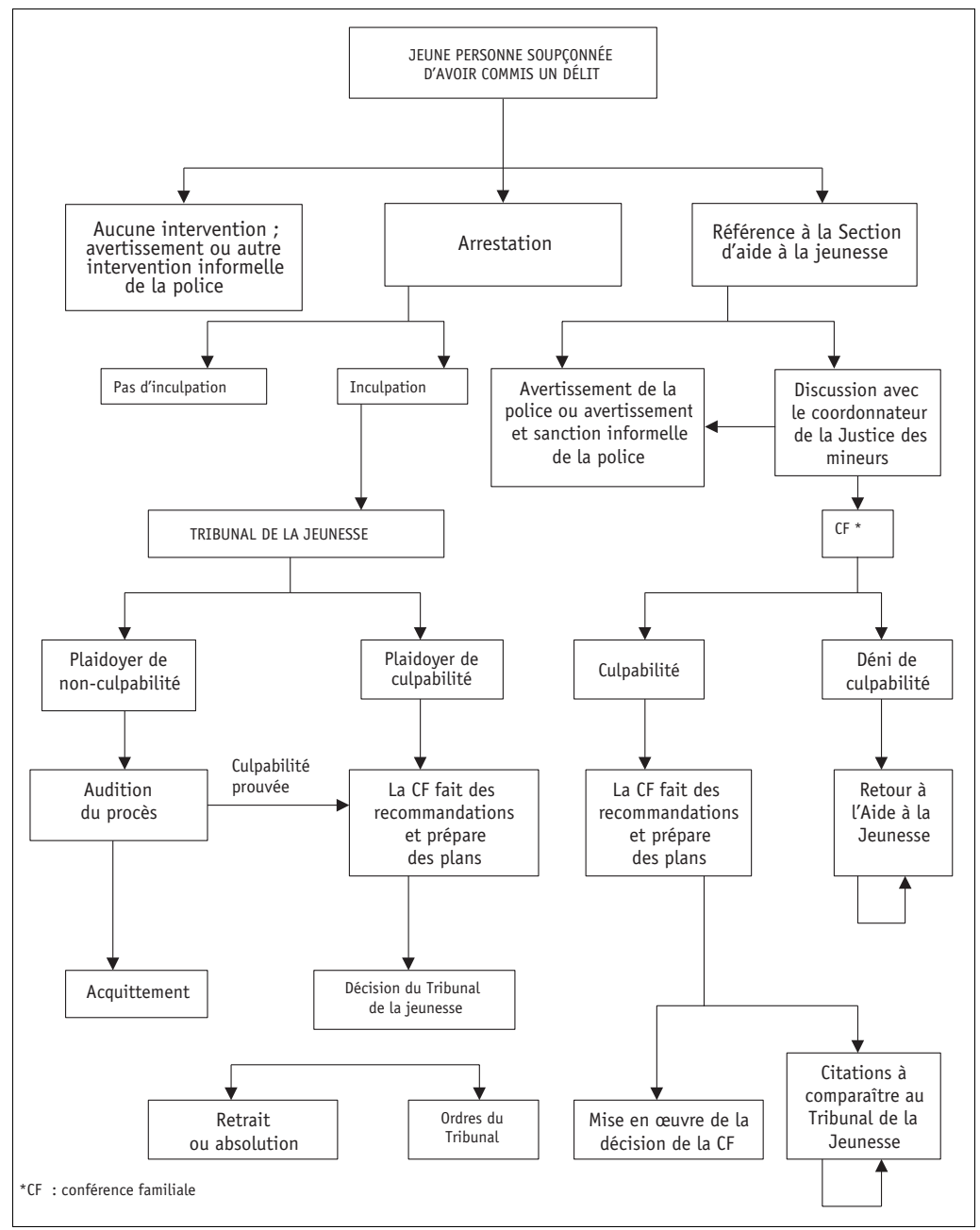

Les corps policiers ont trois possibilités quand ils dépistent de jeunes délinquants : ils peuvent donner un avertissement au jeune et/ou opter pour des sanctions informelles. Ils peuvent aussi confier son cas à une conférence familiale ou procéder à son arrestation. Pour une grande majorité de délinquants (plus de 80 \% en 1996-1997), le traitement de leur dossier se conclut par des avertissements de la police ou par des 
sanctions informelles. Comme c'est le cas maintenant dans la plupart des pays, on s'attend à ce que les jeunes ayant commis des délits mineurs ou ayant contrevenu pour la première fois puissent échapper aux poursuites judiciaires en recevant des avertissements immédiats, " dans la rue ». Quand d'autres démarches sont jugées nécessaires, la police peut, en vue d'assurer un suivi, référer les jeunes à la section d'aide à la jeunesse de la police (une unité spéciale s'occupant seulement des mineurs); cette section peut, par exemple, donner un avertissement en présence des parents. Elle peut aussi exiger que le jeune fasse des excuses à la victime ou imposer des sanctions supplémentaires comme des travaux communautaires. Si la section d'aide à la jeunesse estime qu'elle ne peut s'occuper du délinquant, son cas pourra être référé en vue d'une conférence familiale.

Durant la période 1996-1997, 26 \% des délits portés à la connaissance de la police et attribués à des mineurs ont fait l'objet d'avertissements de la police ; $56 \%$ ont été référés à la section d'aide à la jeunesse et $7 \%$ ont été référés par l'aide à la jeunesse à une conférence familiale. De plus, quelques jeunes délinquants (environ $11 \%$ pendant 19961997) furent arrêtés par la police. Presque tous les jeunes issus de ce dernier groupe furent envoyés par la suite au tribunal de la jeunesse, d'où ils durent être à nouveau référés à des conférences familiales. Le juge du tribunal de la jeunesse ne peut décider d'un cas sans tenir compte des recommandations d'une conférence familiale. On note que, pour l'ensemble des délits commis par des mineurs en 1996-1997, presque un cinquième de ceux-ci a été traité dans le cadre de conférences familiales.

\section{Les conférences familiales}

En Nouvelle-Zélande, les conférences familiales jouent un rôle central au sein du système de justice des mineurs, à la fois pour éviter à des jeunes des poursuites et des sanctions judiciaires et pour décider comment des jeunes délinquants seront traités. Les conférences familiales réunissent plusieurs personnes : le ou la jeune, son avocat (surtout dans les cas référés par le tribunal), les membres de sa famille, la famille élargie (whanau) ou le groupe familial (ceux qui ont une relation de type familial avec la jeune personne), n'importe quelle personne invitée, la ou les victimes ou ceux qui les représentent, ceux qui soutiennent la victime, la police, un médiateur et enfin un travailleur social lorsque le ministère du Bien-Être social a un rôle statutaire en ce qui concerne la 
garde ou la supervision du jeune. D'autres personnes peuvent êtres présentes lors des conférences familiales - par exemple, les frères et sœurs, les amis de la famille, les enseignants, les responsables de groupes de jeunes et ainsi de suite. La participation des victimes revêt une importance particulière et peut avoir des avantages tant pour la ou le jeune, que cela peut aider à évaluer la portée de son délit, que pour la victime, à qui l'on donne la possibilité d'exprimer ses sentiments, de mieux comprendre ce qui s'est passé et d'avoir son mot à dire dans la décision.

Les conférences familiales ne s'occupent que de cas où le mineur a reconnu avoir commis le délit ou en a été reconnu coupable. Une conférence familiale porte essentiellement sur l'acte lui-même et sur les circonstances qui l'ont entouré. Les participants à la conférence élaborent un plan pour le jeune et font des recommandations en vue de le tenir responsable de son délit. Les recommandations offrent un vaste éventail de possibilités : travaux communautaires, travaux pour la victime, réparations matérielles ou excuses, dons à des organismes de charité, restrictions à l'exercice de la liberté du ou de la mineure pour un temps déterminé (assignation à un territoire, couvre-feu, interdiction de conduire une voiture, etc.). Ces recommandations sont limitées seulement par l'imagination des parties. Quand le cas n'est pas passé par le tribunal, la conférence familiale peut encore recommander la poursuite de l'affaire au tribunal et demander à ce dernier d'imposer une mesure particulière. Les décisions sont exécutoires quand tous ceux qui ont participé à la conférence les ont approuvées (et, lorsque c'est requis, quand la décision est approuvée par le juge du tribunal de la jeunesse). Idéalement, le processus à travers lequel le ou la jeune devrait passer inclut les étapes suivantes : l'admission d'avoir mal agi, l'expression de remords, l'engagement de réparer ses torts et la mise au point d'un plan pour prévenir la récidive. Toujours dans l'idéal, la victime devrait pouvoir exprimer sa colère et faire connaître les torts subis ; la situation de la victime devrait être reconnue, elle devrait recevoir réparation et se rétablir.

\section{L'atteinte des objectifs}

Dans cette section, nous nous intéresserons aux objectifs de la justice des mineurs de Nouvelle-Zélande qui sont particulièrement pertinents pour la réforme que propose le gouvernement canadien. 


\section{Tenir les jeunes responsables}

Le gouvernement canadien reconnaît dans son document de stratégie qu'au Canada, le système actuel de justice des mineurs n'a pas réussi à trouver des mesures efficaces et significatives montrant aux jeunes l'effet de leurs actes sur la victime et sur la communauté. Il vise à ce que la stratégie qu'il propose insuffle des valeurs qu'il juge essentielles comme le respect des autres et la responsabilité de ses actes. Le processus judiciaire ne réussit pas particulièrement bien à atteindre de tels objectifs. Par contre, en Nouvelle-Zélande, la plupart des jeunes qui sont pris en charge par le système de justice des mineurs sont tenus responsables en acceptant d'accomplir certaines tâches et/ou en faisant des excuses. Ceci s'applique autant aux cas traités par la police qu'à ceux qui le sont par les conférences et les tribunaux. Par exemple, comme nous l'avons mentionné précédemment, seule une minorité des jeunes traités par la police reçoit un avertissement ; on s'accorde généralement pour recourir à d'autres sanctions. Ainsi, dans l'échantillon de Maxwell et Morris (1993), huit jeunes sur dix parmi ceux qui sont passés par les conférences familiales (incluant ceux qui ont transité par le tribunal de la jeunesse) ont subi ce qu'on appelle des « sanctions actives », c'est-à-dire qu' ils ont fait réparation, qu'ils ont fait des dons, effectué des travaux communautaires ou travaillé pour la victime et/ou subi des restrictions à leur liberté. Quand on ajoute des « excuses » à cette liste, le total atteint $95 \%$. Ainsi, il est rare que les jeunes comptabilisés n'aient « rien à faire ». Signalons d'ailleurs que, dans l'échantillon analysé par Maxwell et Morris (1993), 87 \% des tâches acceptées par les jeunes lors de conférences familiales furent accomplies en entier ou de façon substantielle.

Permettre aux jeunes de prendre part aux décisions relatives aux suites à donner à leur délit constitue une autre manière de les tenir responsables. C'est pourquoi la participation des jeunes délinquants est privilégiée lors des conférences familiales. De ce fait, on s'attend à ce que les jeunes participent directement au processus de prise de décision, qu'ils puissent parler de leur délit et des circonstances qui y sont reliées, qu'ils interagissent avec la victime, qu'ils expriment des remords par rapport à ce qui est arrivé, s'excusent pour ce qu'ils ont fait et prennent part aux décisions concernant les mesures à prendre. La plupart des jeunes qui sont passés par les conférences familiales se sont sentis au moins partie prenante à la décision. Contrairement aux conférences, les tribunaux sont des endroits plus formalistes; on y pratique des rituels étrangers aux jeunes, un langage compliqué, des règles et des procédures 
formelles. Les jeunes ne comprennent souvent pas le déroulement des procédures et y participent rarement de façon active.

\section{Les mesures de rechange}

Actuellement, le Canada recourt beaucoup moins aux mesures de rechange que d'autres pays : $25 \%$ comparé à $53 \%$ aux États-Unis et $57 \%$ en Angleterre et au Pays de Galles. Le document de stratégie du gouvernement fédéral signale que le Canada sur-utilise la mise sous garde pour les auteurs d'infractions non violentes. Pour la majorité de ces jeunes délinquants, le projet fédéral recommande plusieurs alternatives à l'ancien système de justice : avertissements par la police, programmes de mesures de rechange et conférences familiales. De telles méthodes ont bien réussi en Nouvelle-Zélande : les jeunes délinquants ont évité en grand nombre la judiciarisation et l'institutionnalisation. La proportion de jeunes arrêtés est passée d'approximativement un tiers en 1984 à environ $10 \%$ parmi les cas portés à la connaissance de la police. Dans l'ensemble, les comparutions en cour ont beaucoup diminué chez les mineurs - une réduction de presque deux tiers entre 1987 et 1996. De plus, dans l'échantillon de Maxwell et Morris (1993), seulement la moitié des jeunes qui ont comparu devant le tribunal de la jeunesse ont été assujettis à une ordonnance de ce tribunal et seulement $2 \%$ de ces ordonnances concernaient certaines formes de mise sous garde. De façon générale, il y a eu une forte réduction des sentences de mise sous garde pour les jeunes délinquants - une diminution de plus de $50 \%$ entre 1987 et 1996. Ceux qui sont placés sous garde ont, en général, commis des délits très graves (par exemple, vol qualifié avec circonstances aggravantes, viol ou incendie ayant entraîné des dommages importants), ont commis de nombreux délits (un jeune de notre échantillon fut condamné pour 117 chefs d'accusation) ou ont un passé délinquant très chargé. De même, alors qu'avant la loi actuelle les résidences gérées par le ministère du Bien-Être social incluaient 200 places destinées aux jeunes délinquants, elles n'en comptent plus que 76 (bien que des plans visent à augmenter ce nombre à 100). La Nouvelle-Zélande a donc réussi de façon extraordinaire à réduire le nombre de jeunes qui passent en cour et qui sont placés sous garde. Contrairement aux opinions répandues dans le public et les médias, cette réduction n'a pas entraîné une escalade incontrôlée de la délinquance juvénile. S'il est vrai que la délinquance juvénile a augmenté, le taux d'augmentation est demeuré moindre que celui des adultes pour ces dix dernières années. 


\section{S'adapter aux valeurs culturelles}

Les jeunes autochtones sont présents en surnombre dans le système de justice des mineurs au Canada ; la stratégie projetée par le gouvernement reconnaît que toute tentative de traiter la délinquance juvénile doit prévoir des mesures destinées à renforcer les communautés autochtones. La Nouvelle-Zélande a essayé de développer un système de justice qui permette aux différents groupes ethniques de résoudre les problèmes selon des procédures et au moyen de services adaptés à leurs valeurs culturelles. On a cherché à réintroduire des méthodes utilisées par les autochtones pour s'occuper des délinquants - en particulier, on a eu recours à certaines méthodes de résolution des conflits pratiquées traditionnellement dans les familles élargies (whanau $)^{3}$. Cette réintroduction constitue une reconnaissance des obligations contractées dans le traité de Waitangi en 1840, traité qui établissait les bases d'un partenariat entre les Maoris (population indigène de la Nouvelle-Zélande) et les Pakehas; elle reflète aussi l'acceptation progressive de la culture et des valeurs maories au cours de ces quinze dernières années; elle témoigne de la reconnaissance de la pluriethnicité de la population néozélandaise ${ }^{4}$ et dénote aussi une prise de conscience du fait que certains groupes ethniques sont présents en surnombre dans les statistiques criminelles (Statistics New Zealand, 1996).

Les conférences familiales essayent d'accorder une place importante aux procédures de prise de décision; cependant, dans les recherches menées par Maxwell et Morris (1993), on voit que les conférences familiales n'ont souvent pas réussi à intégrer « l'esprit » maori à leurs démarches ni à prendre des décisions en accord avec la philosophie et les valeurs maories. Il arrive que les conférences familiales parviennent à dépasser les apparences et à intégrer un type de processus maori (Maori kaupapa) mais souvent, elles n'y arrivent pas. Une telle démarche suppose la recherche de personnes capables de conseiller et de remettre la responsabilité du processus aux whanau, kapu et iwi, c'est-à-dire à la famille élargie, au clan et à la tribu. De plus, les services iwi (tribaux) et les autorités iwis ne disposent que de peu d'argent ; ces ressources limi-

3. Le Canada connaît une expérience similaire auprès des autochtones avec les cercles de guérison et de sentence. Voir M. Jaccoud, «Les cercles de guérison et les cercles de sentence autochtones au Canada », Criminologie 32 (1) : 79-109. (N.d.É.)

4. Numériquement, le groupe ethnique le plus nombreux en Nouvelle-Zélande est le groupe Pakeha : près de $70 \%$ de la population des jeunes. Les Maoris représentent environ $21 \%$ de la population des mineurs et les Polynésiens $8 \%$. 
tées ne permettent pas de développer une approche réellement culturelle de la justice des mineurs dans la plupart des régions du pays.

Les rituels judiciaires susceptibles d'avoir le plus d'impact sur les délinquants sont ceux qui correspondent à leurs références culturelles. Pour les familles maories, les procédures judiciaires qui intègrent les traditions maories et les traditions occidentales correspondent aux objectifs de la loi et peuvent avoir plus d'impact que si elles obéissaient au seul modèle occidental. Cela se vérifie d'autant plus qu'il existe un protocole de gestion des conflits typiquement maori et largement répandu dans ce groupe. Ce protocole inclut l'expression des remords, envisage les modalités de réparation et permet la prise de décision pour régler le conflit. Suivant le protocole maori, les séances se déroulent en présence des principales parties au conflit. La famille élargie (whanau) doit être présente pour parler au nom de ses jeunes. Permettre ainsi au whanau d'avoir voix au chapitre est très important et ne relève pas d'un «traitement spécial». Bien que les coûts liés à de telles démarches soient plus élevés en termes de temps exigé, le déroulement des séances et l'issue du processus sont susceptibles d'en être améliorés.

Cependant, certains Maoris voudraient que le système de justice subisse des transformations plus radicales. Ils préféreraient que les affaires qui les concernent soient traitées au moyen d'un système de justice exclusivement maories et que les séances se tiennent dans la marae (salle de réunion) (Jackson, 1988). Dans l'ensemble, que l'on préfere des procédures mixtes (maories et occidentales) ou des procédures exclusivement maories, il faut reconnaitre que les processus et les pratiques (et, de ce fait, l'esprit et l'issue des séances) restent aujourd'hui largement inspirés par la culture pakeha, sans considération pour les autres cultures. Malgré cela, les conférences familiales présentent plus de potentiel que les autres types de tribunaux pour faire face à la diversité culturelle.

\section{Encourager la responsabilité des parents}

La nouvelle stratégie canadienne traite peu du rôle des parents. Présentement au Canada, un juge peut exiger la présence d'un parent au tribunal et si ce parent n'obtempère pas, il ou elle peut être accusé d'une infraction. Les nouvelles propositions exigeront en plus que les parents (ou les jeunes eux-mêmes) assument les frais d'avocat quand ils en ont les moyens. Dans cette perspective, la responsabilité parentale est présentée sous un jour négatif, ne considérant que les obligations légales auxquelles les parents doivent se plier. À l'opposé, une approche plus 
positive mettrait l'accent sur le droit des parents de participer et de s'impliquer dans les décisions qui concernent leurs enfants. Dans la nouvelle stratégie canadienne, la seule déclaration qui peut se révéler positive pour les parents est à l'effet que les sentences communautaires seraient employées pour encourager « les membres de la famille...à participer au processus de résolution des conflits et à l'élaboration de solutions face à la délinquance juvénile ».

À l'opposé, les familles néo-zélandaises sont directement impliquées dans la « résolution des conflits et l'élaboration de solutions face à la délinquance juvénile » grâce aux conférences familiales. Elles peuvent y décider du déroulement des séances et des procédures à suivre, elles peuvent choisir les personnes qui seront invitées et le lieu où se tiendra la conférence. Ces responsabilités accordent aux familles un pouvoir à la fois symbolique et pratique. On s'attend aussi à ce que les familles proposent un programme destiné à régler la question du délit.

Les familles participent activement aux conférences en NouvelleZélande, c'est un fait établi. Dans la recherche menée par Maxwell et Morris (1993), presque toutes les conférences comptaient des membres de la famille et deux cinquièmes comptaient aussi des membres de la famille élargie (en ce qui concerne les Maories, la proportion était encore plus forte : presque $60 \%{ }^{5}$ ). Plus des deux tiers des familles interrogées considéraient qu'elles avaient joué un rôle lors des conférences, et à peu près la même proportion considérait qu'elle avait été les décideurs, du moins partiellement. Moins d'un cinquième estimait n'avoir pas été engagé dans le déroulement de l'affaire. $85 \%$ des familles exprimaient aussi leur satisfaction quant à l'issue de la conférence. De ce fait, participer aux conférences rend un certain pouvoir aux familles et leur permet de jouer un rôle primordial lors des décisions à prendre au sujet de leurs enfants, sans pour autant accroître le stigma ou le blâme à leur endroit. De cette manière, « rendre les parents responsables » peut avoir un sens plus positif ${ }^{6}$.

5. Ceci est essentiellement dû au fait que la whanau (famille élargie) joue un rôle plus important dans la société maorie. Traditionnellement, la whanau prenait part à toutes les décisions importantes et à l'éducation des enfants.

6. Il était aussi évident que les familles ayant vécu l'expérience des conférences et des tribunaux préféraient les conférences aux tribunaux. Leurs commentaires mettaient en valeur l'importance que les conférences accordent à la participation de diverses personnes, ils soulignaient aussi le plus grand soutien qui pouvait leur être apporté dans le cadre des conférences familiales tandis que se présenter au tribunal était souvent un facteur de stress. Se sentant plus à l'aise dans les conférences familiales, les familles comprenaient aussi plus clairement ce qui se passait et estimaient que ces conférences constituaient un forum plus adéquat pour prendre des décisions. 


\section{Donner un rôle aux victimes}

Selon l'intention du gouvernement canadien, les victimes devraient être entendues, elles devraient jouer un rôle plus important lors des procédures si elles le désirent et avoir une possibilité de participer à ces dernières. Les mécanismes permettant la réalisation de tels souhaits restent cependant assez flous. Les seuls détails fournis concernent le recours aux sanctions de type communautaire afin de donner aux victimes la possibilité d'exprimer leur point de vue et leurs sentiments et l'implication des victimes dans des programmes de type communautaire comme les conférences familiales et les cercles de sentence. Cependant, il n'y a pas d'indications sur la proportion des cas pouvant faire l'objet de telles procédures ou sur leur niveau de gravité. Comme nous le faisions remarquer précédemment, les propositions canadiennes considèrent encore les tribunaux comme des éléments-pivots du système, spécialement quand il s'agit de traiter des délits plus graves, c'est-à-dire dans ces cas où les victimes pourraient le plus bénéficier de ces autres façons de faire.

En Nouvelle-Zélande, au contraire, les victimes peuvent participer aux processus de la justice des mineurs. Elles peuvent intervenir lorsque la police décide de mesures de rechange (elles sont consultées sur ce qui devrait arriver au jeune et peuvent accepter que celui-ci accomplisse des travaux pour elles) ; plus spécifiquement, les victimes peuvent aussi participer aux conférences familiales qui, nous l'avons déjà mentionné, s'occupent d'affaires moyennement graves ou graves. Les victimes ont le droit d'assister aux conférences. Même si les recherches faites (Maxwell et Morris, 1993) ont constaté que les victimes n'étaient présentes que dans la moitié des cas étudiés, il semble que cette situation soit principalement due à une mauvaise gestion des rencontres. Certes, il y aura toujours une minorité de victimes qui choisiront de ne pas participer aux conférences ; la recherche menée en Nouvelle-Zélande a néanmoins découvert que seulement $6 \%$ des victimes interrogées affirmaient ne pas vouloir rencontrer leur agresseur.

La recherche menée en Nouvelle-Zélande a aussi montré que, quand les victimes avaient participé à ces conférences, un grand nombre d'entre elles considéraient cette démarche sous un jour positif. Environ $60 \%$ des victimes interrogées qualifiaient ces conférences d'utiles, de positives et de gratifiantes. En général, elles considéraient qu'elles avaient participé activement au processus et qu'elles se sentaient mieux après avoir ainsi participé. Les victimes mentionnaient aussi deux autres effets 
bénéfiques. D'abord, elles avaient pu faire entendre leur voix lors du choix des solutions. Ensuite, elles avaient pu rencontrer face à face l'agresseur et sa famille, de sorte qu'elles avaient pu mieux comprendre leur attitude, saisir les raisons du délit et évaluer les probabilités de récidive. Environ un quart des victimes interrogées ont affirmé se sentir plus mal à l'issue des conférences. Cependant, leur insatisfaction était causée par la mauvaise gestion de la conférence et non par le principe de la conférence elle-même.

Un pourcentage significatif de victimes se montrait aussi satisfait de l'issue des conférences. Comme nous l'avons écrit précédemment, les conférences menaient surtout à des excuses aux victimes par les jeunes ou à des travaux communautaires (au bénéfice de la victime ou d'une organisation désignée par cette dernière) ; les réparations directes à la victime étaient moins souvent exigées. Certaines victimes restaient insatisfaites parce qu'elles jugeaient les sanctions trop douces ou trop dures. Plus souvent, les victimes se montraient insatisfaites parce que les arrangements convenus ne s'étaient pas concrétisés par la suite ou parce qu'elles n'avaient jamais été informées des résultats finaux de la conférence. De telles situations sont le plus souvent dues aux professionnels et non au jeune et à sa famille. Dans l'ensemble, les victimes étaient moins satisfaites de l'issue des conférences que les professionnels et les familles. Cependant, ce taux de satisfaction est presque certainement plus élevé que celui que les victimes exprimeraient après des auditions en cour et les sentences des juges.

\section{Récidives et conférences familiales}

Un des objectifs principaux de la stratégie que propose le gouvernement canadien est la protection de la société grâce à la réduction de la délinquance juvénile. Il existe beaucoup de controverse concernant les capacités préventives du système de justice traditionnel. En ce qui concerne les conférences familiales, de récentes recherches ont au contraire fourni des résultats encourageants en matière de prévention de la récidive. Nous avons ainsi fait le suivi d'un échantillon de jeunes ayant pris part à des conférences familiales en 1996-1997 ; nous les avons interrogés et avons interrogé leurs parents. Nous tentions d'identifier les facteurs distinguant les jeunes qui avaient été déclarés coupables d'infractions d'autres jeunes qui ne l'avaient pas été 7 . Sur la base d'analyses à plusieurs variables, nous avons trouvé que les facteurs de prédiction de la récidive étaient reliés aux expériences datant de la petite enfance, à l'histoire criminelle et à des 
événements subséquents de la vie des jeunes. Les facteurs liés aux conférences familiales avaient aussi une grande influence.

Nous avons aussi demandé aux jeunes et à leurs parents s'ils avaient été d'accord avec les décisions prises lors des conférences et s'ils s'étaient sentis impliqués dans le processus. L'analyse des réponses montre que ceux qui étaient en accord avec les décisions et qui s'étaient sentis impliqués étaient plus susceptibles de compter parmi les non-récidivistes que parmi les récidivistes persistants. Nous avons aussi interrogé les jeunes sur les remords et la honte qu'ils auraient pu éprouver. L'analyse a démontré que, lorsque les sentiments de honte perduraient, le pronostic n'avait pas tendance à être favorable. D'autre part, si l'on définit le remords comme le fait de se sentir désolé de ses actes, d'accomplir des tâches et de tenter de réparer ses torts ${ }^{8}$, la présence de remords était plutôt associée avec un retrait de la délinquance.

En résumé, les points suivants sont des facteurs de prédiction significatifs de non-récidive :

Pour les parents :

- avoir des remords ;

- ne pas éprouver de honte parce qu'on les a considérés comme de mauvais parents ;

- trouver des solutions justes et les accepter.

Pour les jeunes :

- être d'accord avec les décisions de la conférence familiale ;

- avoir des remords ;

- ne pas éprouver de honte alors que les autres tentent de nous amener à nous sentir mal ;

- se sentir impliqué lors des prises de décision des conférences familiales.

Les résultats de cette étude apportent donc des éléments de preuve à l'effet que, quand elles sont bien organisées, les conférences familiales peuvent avoir une influence sur les comportements délinquants à venir.

7. Sur base de données de délinquance auto-révélée, nous avons pu établir que les personnes qui n'avaient pas été déclarées coupables étaient en liberté parce qu'elles n'avaient commis aucun délit et non parce qu'elles avaient évité le dépistage des policiers.

8. Notre définition du remords se fonde sur la somme d'un nombre d'éléments dérivés d'une analyse factorielle qui produisit une composante principale faite des réponses « oui » ou « non » des jeunes aux questions suivantes : se souvenaient-ils de la conférence? Avaientils accompli les tâches convenues? Regrettaient-ils leurs actes et l'avaient-ils montré? Estimaient-ils avoir réparé leurs torts? Du point de vue des parents, on estimait qu'ils avaient éprouvé des remords si les parents répondaient « oui » à la question suivante : «Pensez-vous que votre fils ou votre fille regrettait ce qu'il ou elle avait fait? » 


\section{Conclusions}

Les propositions canadiennes poursuivent divers objectifs. Cependant, il n'est pas clair que les moyens choisis permettront de réaliser ces objectifs. En Nouvelle-Zélande, beaucoup d'objectifs prévus ont déjà été atteints. Ainsi, depuis les réformes de 1989, les jeunes contrevenants sont moins nombreux à passer en cour et à être mis sous garde. De même, presque tous les jeunes qui ont commis des délits suffisamment graves pour justifier l'organisation d'une conférence familiale sont tenus responsables de leurs infractions. Les coutumes des différents groupes culturels ont aussi été prises en compte et, dans certains cas, on a utilisé des méthodes traditionnelles pour parvenir à un accord. Enfin, il semble que des conférences familiales bien organisées contribuent à la prévention de la récidive.

Bien qu'il existe des variations significatives entre provinces, on reconnait au Canada que l'on surutilise la mise sous garde pour les jeunes délinquants. Il faut s'interroger sérieusement sur les capacités de la stratégie proposée à juguler cet abus. Multiplier les alternatives à l'enfermement s'est révélé maintes et maintes fois inefficace : les solutions de rechange de type communautaire se substituent simplement les unes aux autres. La stratégie gouvernementale propose également le recours à l'humiliation publique et aux sentences pour adultes à l'endroit des délinquants graves et multirécidivistes. Cependant, il n'y a pas de raisons de penser que de telles mesures auront des effets positifs sur les jeunes, les victimes et les communautés impliquées. En fait, on pourrait obtenir de meilleurs résultats en informant le public sur la réalité de la délinquance juvénile et son peu d'importance relative quand on la compare à la criminalité adulte; en responsabilisant les jeunes de manière significative ; en travaillant activement auprès des victimes afin de répondre à leurs besoins et d'assurer leur sécurité future ; en consolidant et en appuyant les familles qui éprouvent des difficultés avec leurs enfants et en aidant les communautés à développer des stratégies constructives pour réagir à la délinquance.

\section{Références}

Braithwaite, J. 1989. Crime, Shame and Reintegration, Cambridge : Cambridge University Press.

JACKSON, M. 1988. The Maories and the Criminal Justice System, Part II, Wellington,

New Zealand : Department of Justice. 
Maxwell, G. M. 1998. Researching Reoffending, document inédit présenté à la conférence « Youth Justice in Focus », Wellington, New Zealand.

MaXwell, G. M. et MORRIS, A. 1993. Family, Victims and Culture : Youth Justice in New Zealand, Wellington, New Zealand : Social Policy Agency and Institute of Criminology, Victoria University.

MCGuire, J. (dir.). 1995. What Works: Reducing Reoffending, Chichester, England : Wiley.

Ministère de la Justice. 1998. Stratégie de renouvellement du système de justice pour les jeunes, Ottawa : Ministère de la Justice du Canada.

Morris, A., Maxwell, G. M. et HoOper, R. 1998. Preliminary Report on Understanding Reoffending, rapport inédit, Institute of Criminology : Victoria University of Wellington.

Statistics New Zealand. 1996. New Zealand Crime Now, Wellington, New Zealand : Statistics New Zealand.

Tangney, J. P., Wagner, P. E., Hill-Barlow, D., Marschall, D. E. et Gramzow, R. 1996. «Relation of Shame and Guilt to Constructive versus Destructive Responses to Anger across the Lifespan », Journal of Personality and Social Psychology 70 (4) : 797-809.

Thomson, G. 1998. «Youth Justice Renewal », p. 105-107 in : Youth Justice Conference: An International Perspective on Problems and Solutions: Conference Proceedings, Calgary : Canadian Research Institute for Law and Family. 